\title{
UKRN Position on COVID-19 Research
}

The UK Reproducibility Network Steering Group

$1^{\text {st }}$ May 2020

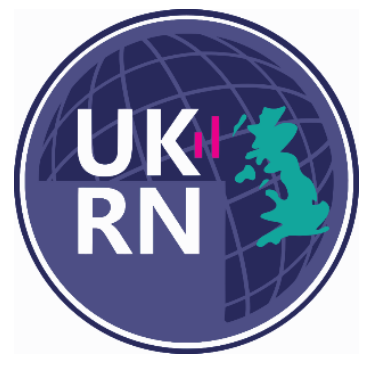

Fast, cheap, good - you can pick two...

The UK Reproducibility Network (UKRN) is a peer-led consortium that aims to ensure the UK retains its place as a centre for world-leading research. The UKRN grew from activity across the UK seeking to understand the factors that contribute to poor research reproducibility and replicability. We aim to develop approaches to counter these problems, and thereby improve the quality of the research we produce.

The COVID-19 pandemic is unprecedented in recent history, and has demonstrated the strength of the global scientific community. Resources have been rapidly diverted towards understanding the virus, modelling strategies to reduce its impact, developing vaccines and treatments, and more. Collaborations - both national and international - have emerged almost overnight, and preprint servers have experienced a surge of submissions.

However, given the importance and the immediacy of the challenge, rigorous and high-quality research is more important than ever. There is an urgent need for data and knowledge, but it is critically important that data are of high quality and that knowledge truly advances: false information is worse than no information.

Open research can serve as a quality-control process, protecting against bias, minimising errors in the research process, and providing greater scope for errors to be detected by the wider community. To promote the uptake of open research practices, we have produced a series of primers - on pre-registration, data sharing, open code and software, open access, and preprints.

There are also challenges for research users in assimilating and critically appraising the findings of the large number of scientific publications and preprints, so that these might be deployed for the public good. We therefore welcome the collaborative engagement of the global systematic review community, and encourage them to further strengthen these collaborations to enhance the efficiency and timeliness of their work.

In any human endeavour errors will occur - scientists are not infallible. And this will be particularly true when working fast or under time pressure. The fundamentals of good design, careful conduct and thoughtful interpretation apply even when there is a pressing need to understand a new phenomenon rapidly. But in addition to this, transparency is a vital - to ensure that work can be scrutinised and trusted.

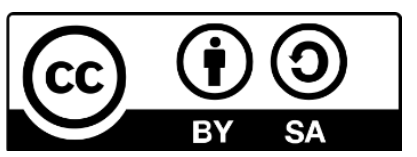

This work is licensed under a Creative Commons Attribution-ShareAlike 4.0 International License. 